University of New Hampshire

University of New Hampshire Scholars' Repository

Physics Scholarship

Physics

6-1994

\title{
COMPTEL imaging of the Galactic disk and the separation of diffuse emission and point sources
}

\author{
H Bloemen \\ Space Research Organization of the Netherlands \\ W Hermsen \\ Space Research Organization of the Netherlands (SRON) \\ B Swanenburg \\ SRON \\ C de Vries \\ SRON \\ R Diehl \\ Max-Planck-Institut für extraterrestriche Physik

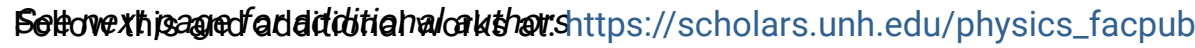 \\ Part of the Astrophysics and Astronomy Commons \\ Comments \\ For copyright information http://www.adsabs.harvard.edu/
}

\section{Recommended Citation}

H. Bloemen, W. Hermsen, B. N. Swanenburg, C. P. de Vries, R. Diehl, V. Schoenfelder, H. Steinle, A. W. Strong, A. Connors, M. McConnell, D. Morris, G. Stacy, K. Bennett, and C. Winkler, 'COMPTEL imaging of the Galactic disk and the separation of diffuse emission and point sources', The Astrophysical Journal Supplement Series, vol. 92, p. 419, Jun. 1994.

This Article is brought to you for free and open access by the Physics at University of New Hampshire Scholars' Repository. It has been accepted for inclusion in Physics Scholarship by an authorized administrator of University of New Hampshire Scholars' Repository. For more information, please contact Scholarly.Communication@unh.edu. 


\section{Authors}

H Bloemen, W Hermsen, B Swanenburg, C de Vries, R Diehl, V. Schonfelder, H Steinle, A W. Strong, A Connors, Mark L. McConnell, D Morris, G Stacy, K Bennett, and C Winkler 
The ASTROPHYSICAL Journal SUPPLEMENT SERIES, 92:419-423, 1994 June

(9) 1994. The American Astronomical Society. All rights reserved. Printed in U.S.A.

COMPTEL IMAGING OF THE GALACTIC DISK AND THE SEPARATION OF DIFFUSE EMISSION AND POINT SOURCES

\author{
H. Bloemen, ${ }^{1}$ W. Hermsen, B. N. Swanenburg, and C. P. DE VRIES \\ SRON-Leiden, P.O. Box 9504, 2300 RA Leiden, The Netherlands \\ R. DieHL, V. SCHÖNFELDER, H. STEINLE, AND A.W. STRONG \\ Max-Planck-Institut für Extraterrestrische Physik, D-8046 Garching, Germany
}

A. CONNORS, M. MCCONNELl, D. MORRIS, AND G. STACY ${ }^{2}$

Space Science Center, University of New Hampshire, Durham, NH 03824

AND

K. BENNETT AND C. WINKLER

Astrophysics Division, European Space Research and Technology Centre, 2200 AG Noordwijk, The Netherlands

Received 1993 April 12; accepted 1993 August 12

\begin{abstract}
The first MeV image of the inner Galaxy obtained with the COMPTEL telescope aboard the Compton GammaRay Observatory shows a structured ridge of emission along the Galactic equator which probably consists of diffuse radiation and unresolved discrete sources. The intensity distribution of this ridge distinctly differs from the expected distribution for a simple model of the diffuse radiation. Most remarkable are the observed excursions out of the disk, which may hint at the presence of $\gamma$-ray point sources located several degrees away from the mid-plane, but a diffuse origin cannot be excluded. If these are indeed discrete sources, a substantial fraction of the unresolved emission along the disk may not be of diffuse origin, with an ensemble of sources hidden in the observed ridge.
\end{abstract}

Subject headings: diffuse radiation — gamma-rays: observations

\section{INTRODUCTION}

The COMPTEL telescope aboard the Compton GammaRay Observatory provides for the first time extensive imaging possibilities at $\mathrm{MeV}$ energies with a source-location accuracy of typically $1^{\circ}$. This imaging capability is of particular value for studies of the Galactic disk, where a variety of discrete sources as well as diffuse emission can be expected to contribute. The radiation at energies above $\sim 50 \mathrm{MeV}$, well studied with the $S A S 2$ and $\operatorname{COS} B$ satellites, seems largely of diffuse origin (see, e.g., review by Bloemen 1989). Unresolved point sources may be hidden, but preliminary results from EGRET indicate that the diffuse emission plays indeed a dominant role. Below $\sim 50 \mathrm{MeV}$, current knowledge is limited because the observations are difficult and have sofar mainly been made with nonimaging instruments with fields of view of typically tens of degrees. Figure 1 shows a compilation of flux estimates for the central radian of the Galactic disk. A detailed comparison is not possible because of differences in the longitude intervals covered by the observations and differing assumptions in calculating the flux from the central radian of the Galaxy. Nevertheless, above $\sim 3 \mathrm{MeV}$, all measurements are consistent, with the possible exception of the $S M M$ spectrum presented by Harris et al. (1990). Around $1 \mathrm{MeV}$ (between the positron annihilation line at $511 \mathrm{keV}$ and $\sim 3 \mathrm{MeV}$ ), however, significant differences are seen, which may be real. Most remarkable is the drastic flux decrease between the two HEAO3 observa-

\footnotetext{
${ }^{1}$ Also Leiden Observatory, The Netherlands.

${ }^{2}$ Also Compton Observatory Science Support Center, NASA GSFC.
}

tions of the Galactic center region (Riegler et al. 1985), which cover a field of view as wide as $\sim 35^{\circ}$ (only the "low-state" upper limit is included in Fig. 1-the high-state flux is more than an order of magnitude higher). This suggests a strong contribution from one or two point sources; the high-state spectrum between $\sim 100 \mathrm{keV}$ and several $\mathrm{MeV}$ obtained by Riegler et al. is very similar to that of the blackhole candidate Cyg X-1 during its $\gamma_{1}$ phase (Ling et al. 1987). Also at 100 $\mathrm{keV}$, the observed radiation from the central $20^{\circ}$ of the Galactic disk is known to be variable-most of the emission can be attributed to two sources, 1E 1740.7-2942 and GRS 1758-258 (see review by Gehrels \& Tueller 1993).

This paper describes our analysis strategy to map the observed emission and to disentangle the contributions from point sources and diffuse emission. Preliminary results are presented for a combination of 10 observations of the first and fourth Galactic quadrants, obtained during the first half of the COMPTEL all-sky survey (Table 1). First results were already given by Bloemen et al. (1993). Because of uncertain spectral characteristics, all analyses presented here were applied to three separate energy intervals, $0.75-1 \mathrm{MeV}, 1-3 \mathrm{MeV}$, and 3-10 MeV, but the findings have been combined. Spectral variations will be addressed in future work.

\section{METHODS OF IMAGING AND SEARCH FOR SOURCES}

Each incident photon detected by COMPTEL is first Compton scattered in the upper layer of detectors, $D 1$, and then absorbed in the lower layer, $D 2$ ( see Schönfelder et al. 1994 for a description of the instrument). The energy deposits in the two layers, $E_{1}$ and $E_{2}$, define the energy of the incident photon, 


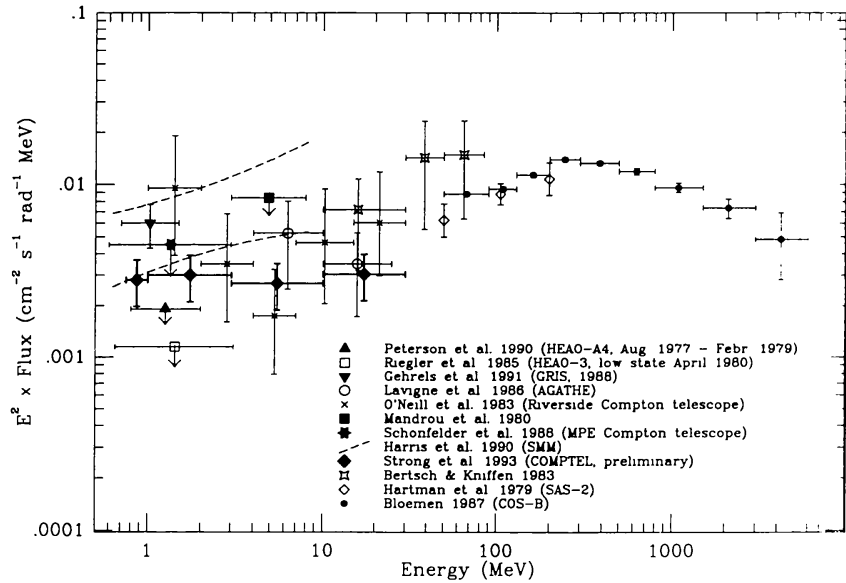

FIG. 1.-Compilation of flux estimates for the inner Galaxy, multiplied by $E^{2}$. An $E^{-2}$ spectrum was assumed to calculate effective energies. The published fluxes from $H E A O 3$ and GRIS were converted to flux-perradian by dividing by the FWHM of the instrument field of view. The preliminary COMPTEL data points are taken from Strong et al. in this volume.

$E_{\gamma} \equiv E_{1}+E_{2}$, as well as the Compton scatter angle, $\bar{\varphi} \equiv \arccos$ $\left(1-m_{e} c^{2} / E_{2}+m_{e} c^{2} / E_{\gamma}\right)$. The interaction locations define the scatter direction. For a selected energy range, the photons can thus be binned in a three-dimensional data space consisting of the scatter direction, hereafter denoted by the arbitrary coordinates $\chi$ and $\psi$, and the scatter angle, $\bar{\varphi}$. The search for sources, model fitting, etc., are all carried out in this three-dimensional data space with a typical $\chi, \psi, \bar{\varphi}$ binning of $1^{\circ} \times$ $1^{\circ} \times 2^{\circ}$

For a given $\gamma$-ray intensity distribution, $I(\chi, \psi)$, the expected number of photons in a data space cell is in good approximation given by

$$
\begin{aligned}
N(\chi, \psi, \bar{\varphi})= & G(\chi, \psi, \bar{\varphi}) \iint \chi_{\chi^{\prime}, \chi^{\prime}} d \Omega d \chi^{\prime} d \psi^{\prime} \\
& \times I\left(\chi^{\prime}, \psi^{\prime}\right) A\left(\chi^{\prime}, \psi^{\prime}\right) f\left(\chi^{\prime}-\chi, \psi^{\prime}-\psi, \bar{\varphi}\right),
\end{aligned}
$$

where $d \Omega$ is the solid angle covered by the bin at $(\chi, \psi), A$ is the effective exposure of the $D 1$ layer $\left(\mathrm{cm}^{2} \mathrm{~s}\right), f$ is the three-dimensional point-spread function (PSF), defined for an infinite

TABLE 1

COMPTEL ObSERVATIONS USED IN PRESENT ANALYSIS

\begin{tabular}{lrrcc}
\hline \hline & \multicolumn{2}{c}{ POINTING } & & \\
\cline { 2 - 3 } OBSERVATION & \multicolumn{1}{c}{$l$} & \multicolumn{1}{c}{$b$} & START & END \\
\hline $2.0 \ldots \ldots \ldots \ldots$ & 73.3 & +2.6 & $1991-05-30$ & $1991-06-08$ \\
$5 \ldots \ldots \ldots \ldots$ & 0.0 & -4.0 & $1991-07-12$ & $1991-07-26$ \\
$7.0 \ldots \ldots \ldots$ & 70.4 & -8.3 & $1991-08-08$ & $1991-08-15$ \\
$7.5 \ldots \ldots \ldots \ldots$ & 25.0 & -14.0 & $1991-08-15$ & $1991-08-22$ \\
$12 \ldots \ldots \ldots \ldots$ & 310.7 & +22.2 & $1991-10-17$ & $1991-10-31$ \\
$13.0 \ldots \ldots \ldots$ & 25.0 & -14.0 & $1991-10-31$ & $1991-11-07$ \\
$14^{a} \ldots \ldots \ldots \ldots$ & 285.0 & -0.7 & $1991-11-14$ & $1991-11-28$ \\
$16 \ldots \ldots \ldots \ldots$ & 0.0 & +20.2 & $1991-12-12$ & $1991-12-27$ \\
$20^{\mathrm{a}} \ldots \ldots \ldots \ldots$ & 39.7 & +0.8 & $1992-02-06$ & $1992-02-20$ \\
$23^{\mathrm{a}} \ldots \ldots \ldots \ldots$ & 322.1 & +3.0 & $1992-03-19$ & $1992-04-02$ \\
\hline
\end{tabular}

${ }^{a}$ Only part of the available data included here. extent of the $D 2$ layer (a perfect cone if the energy and position determinations in $D 1$ and $D 2$ were exact), and $G$ is the probability that a photon scattered in $D 1$ in a direction $(\chi, \psi)$ indeed encounters a $D 2$ module (a purely geometrical function). The latter also includes the effect of cuts in the data space which are needed when the Earth is in the field of view. Including this effect introduces the $\bar{\varphi}$ dependence of $G$. The response of the instrument is known from pre-launch calibration and simulations. The art of imaging with COMPTEL involves recognizing the ( $G$-modified) cone-shaped patterns in the data space. Two main techniques are applied: a maximum-entropy method for general imaging purposes (Strong et al. 1992) and a likelihood method optimized to search for point sources (de Boer et al. 1992), including the determination of the statistical significance, flux, error region, etc. This paper presents results from both mapping methods. In addition, likelihood optimization is used for model fitting, as described in $\S 4$.

\section{BACKGROUND MODELING}

Preliminary studies of the COMPTEL data have shown that typically $\sim 95 \%$ of the number of observed $\gamma$-rays should be attributed to the background (instrumental and isotropic). The likelihood analyses in particular require a careful estimate of this background, for which a variety of methods is being studied. The background models used in the present work are determined by applying a filter technique to the three-dimensional data space, which smooths the photon distribution and eliminates source signatures in first-order approximation. The likelihood algorithms for source mapping and model fitting use a modified instrument response to take this filtering into account. For point sources and line sources, simulations have shown that this method cannot be significantly improved upon by using independent background estimates.

Figure 2 presents a one-dimensional illustration of the filter principle for a source signal on a flat background. Basically, for
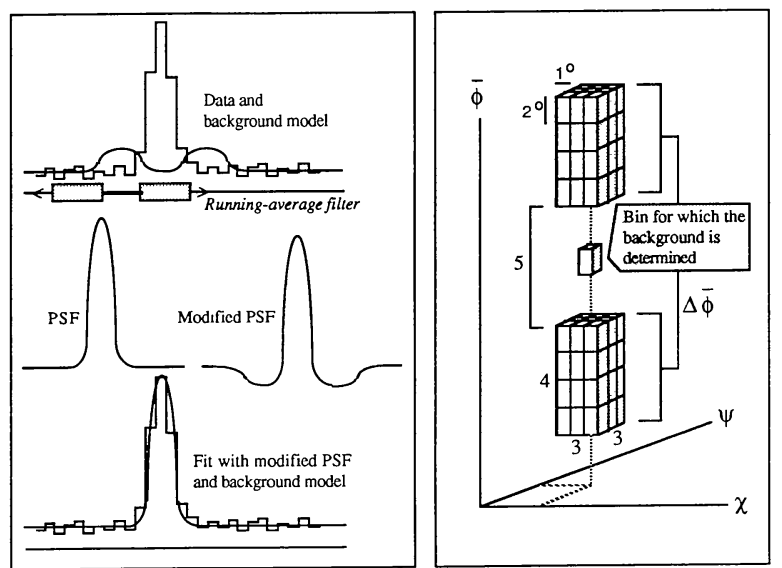

FIG. 2.- (left) Simplified one-dimensional illustration of the filter technique for background modeling applied in this paper, together with the corresponding response modification. Note the presence of the two source remnants in the background model and the compensating negative side lobes of the modified PSF. ( right) Illustration of the bin selection for profile fitting in the three-dimensional COMPTEL data space. The indicated numbers/sizes were found to be optimal independent of $\gamma$-ray energy and are used for all analyses presented here (but further optimization may be possible). 
this simple case, the background model is defined as the running average over a selected range of bins, excluding the central part of this range (although this is not necessary); the source is removed, but two adjacent source remnants are created. The same averaging is applied to the PSF and the result is subtracted from the PSF, which introduces negative side lobes that compensate for the source remnants in the background model. In the COMPTEL data space, this filter technique is applied in the $\bar{\varphi}$ direction, with the exception that instead of a running average a profile is fitted over the selected range of bins. This is necessary because the background has a broad bump shape in $\bar{\varphi}$ direction. The key finding that has led to the filter method is the observation that this shape (after correction for the cuts that are made to exclude the Earth in the field of view- $\$ 2$ ) is globally independent of $\chi, \psi$, which implies that the fit profiles can be derived from the observations. For the interested reader, we summarize here the technical details. The three-dimensional background matrix is defined by

$$
B(\chi, \psi, \bar{\varphi})=P(\chi, \psi, \bar{\varphi}) \frac{\sum_{\Delta \bar{\varphi}^{\prime}}\left\langle N\left(\chi, \psi, \bar{\varphi}^{\prime}\right)\right\rangle}{\sum_{\Delta \bar{\varphi}^{\prime}} P\left(\chi, \psi, \bar{\varphi}^{\prime}\right)},
$$

where the profile matrix $P$ that is fitted (normalized) here to the photon distribution is equal to the geometry matrix $G$, normalized to the number of observed photons for a given $\bar{\varphi}$,

$$
P(\chi, \psi, \bar{\varphi})=G(\chi, \psi, \bar{\varphi}) \frac{\sum_{\chi^{\prime}, \psi^{\prime}} N\left(\chi^{\prime}, \psi^{\prime}, \bar{\varphi}\right)}{\sum_{\chi^{\prime}, \psi^{\prime}} G\left(\chi^{\prime}, \psi^{\prime}, \bar{\varphi}\right)}
$$

The quantity $\Delta \bar{\varphi}$ in equation (2) indicates the range of $\bar{\varphi}$ bins selected for the fitting, as illustrated in Figure 2: a selection of 13 bins in $\bar{\varphi}$ direction, $2^{\circ}$ wide, appears to be optimal, of which the central five bins are excluded. In order to reduce statistical fluctuations in the background model, a weak smoothing in $\chi$, $\psi$ direction is applied ( $3 \times 3$ bins) prior to the fitting, as indicated by the \langle\rangle brackets in equation (2) (and eq. [5] below), i.e., $(13-5) \times 3 \times 3=72$ bins are involved in the fitting for each point of the data space. During the likelihood scanning, the effective PSF at each scan position $\chi_{0}, \psi_{0}$, which is given by

$$
F_{\chi 0, \psi_{0}}(\chi, \psi, \bar{\varphi}) \equiv f_{\chi_{0}, \psi_{0}} \times G(\chi, \psi, \bar{\varphi}),
$$

is modified accordingly:

$$
\begin{aligned}
F_{\chi_{0}, \psi_{0}(\chi, \psi, \bar{\varphi})}^{\prime}=F_{\chi_{0}, \psi_{0}}(\chi, \psi, \bar{\varphi}) & \\
& -P(\chi, \psi, \bar{\varphi}) \frac{\sum_{\Delta \bar{\varphi}^{\prime}}\left\langle F_{\chi_{0}, \psi_{0}}\left(\chi, \psi, \bar{\varphi}^{\prime}\right)\right\rangle}{\sum_{\Delta \bar{\varphi}^{\prime}} P\left(\chi, \psi, \bar{\varphi}^{\prime}\right)} .
\end{aligned}
$$

Sky models (e.g., diffuse emission) fitted to the data space are modified in a similar way.

The likelihood maps presented in the present paper show the quantity $-2 \ln \lambda$, where $\lambda$ is the maximum likelihood ratio $L$ (background) $/ L$ (source + background). Formally, in a search for sources, $-2 \ln \lambda$ has a chi-square distribution with 3 degrees of freedom (for instance, a $3 \sigma$ detection corresponds to $-2 \ln \lambda=13.8$ ). Since we combine here the results for three separate energy ranges (the maps of $-2 \ln \lambda$ shown are the sum of three maps), $-2 \ln \lambda$ has a chi-square distribution with 5 degrees of freedom in a search for sources (a $3 \sigma$ detection corresponds to $-2 \ln \lambda \simeq 18$ in this case). Simulations have shown, however, that our method of background modeling requires a somewhat more stringent criterion for source detection. The main reason for this is in the source remnants included in the background model, which introduce a dependency of source and background (the "artifacts" added to the background suppress the likelihood of the null hypothesis, but are then perfectly compensated with the inverse artifacts-the negative side lobes of the PSF-in the alternative hypothesis). We plan to further optimize this method.

\section{MODEL FITTING AND SIMULATIONS OF DIFFUSE EMISSION}

In order to verify the imaging potential of COMPTEL for complex regions and to compare the observations with the results expected from diffuse emission, we also applied our mapping tools to Monte Carlo simulations of a model of the diffuse (bremsstrahlung) emission. This simple model is based on the assumption that the cosmic-ray electron density is constant throughout the Galaxy, so the $\gamma$-ray intensity distribution is approximately given by $I_{\gamma}=\epsilon_{\gamma} N_{\mathrm{H}}$, where $N_{\mathrm{H}}$ is the columndensity distribution of the interstellar gas, derived from various $\mathrm{H}$ I surveys and the $\mathrm{CO}$ survey by Dame et al. (1987). A CO-to- $\mathrm{H}_{2}$ conversion factor of $2.3 \times 10^{20}$ molecules $\mathrm{cm}^{-2}$ $\left(\mathrm{K} \mathrm{km} \mathrm{s}^{-1}\right)^{-1}$ was adopted (Strong et al. 1988); for the strong $\mathrm{CO}$ emission from the Galactic center, the conversion factor was assumed to be 10 times lower (Blitz et al. 1985). First, the value of the $\gamma$-ray emissivity, $\epsilon_{\gamma}$, was determined for each energy interval by maximum-likelihood fitting of the model to the binned photons in the three-dimensional data space. The values are consistent with the results of Strong et al. (this volume), using a somewhat different subset of COMPTEL observations and high-latitude observations for the background modeling. Using these emissivities, simulated three-dimensional dataspaces of the diffuse emission were made, to which background simulations were added. This exercise was done twice in order to illustrate the influence of statistics on the resulting images.

\section{RESULTS AND CONCLUSIONS}

Figure 3 shows our mapping results in the energy range $0.75-10 \mathrm{MeV}$ (a combination of the results for the three separate energy ranges: $0.75-1,1-3$, and 3-10 MeV), both for the actual observations ( right) and for the two independent simulations of the diffuse emission. The likelihood maps and maximum-entropy maps are very similar, the latter showing more detailed structure because of the deconvolution inherent in the maximum-entropy method. The global appearance of the two simulations is similar, as expected, although small-scale differences can be seen, illustrating the impact of limited statistics.

The observations clearly show a ridge of emission, but in detail distinctly different from the simulations. Its wobbling appearance (with evidence for spectral variations, to be addressed in future work) has been puzzling from the start and only very recently gained confidence with the COMPTEL imaging results of the $1.809 \mathrm{MeV}$ line from the decay of interstellar ${ }^{26} \mathrm{Al}$. The maximum-entropy and likelihood maps in the 1.7-1.9 MeV band (Fig. 4-from Diehl et al. 1994), obtained with precisely the same methods as described here (implying that any continuum emission is included), do show a narrow 

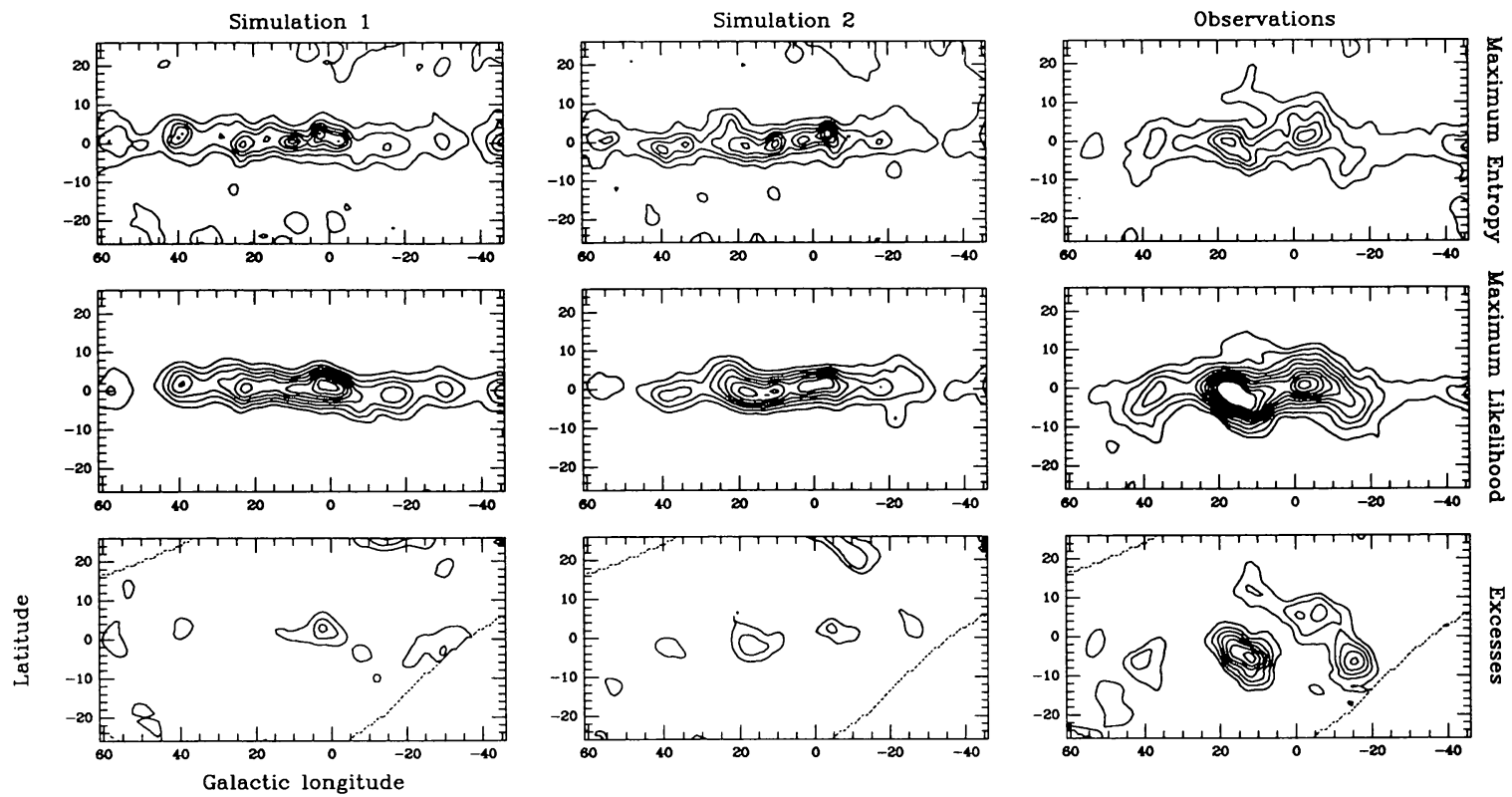

FIG. 3.-Maximum-entropy maps and maximum-likelihood maps of combined COMPTEL observations of the inner Galaxy (0.75-10 MeV, Table 1) and two simulated observations of the diffuse emission, with emissivity from model fitting. Contour levels: (top) start $5 \times 10^{-3}, \mathrm{step}^{3} \times 10^{-3} \mathrm{~cm}^{-2} \mathrm{~s}^{-1} \mathrm{sr}^{-1}$; (middle) start 15, step 15; (bottom) start 15, step 10. See text for further details.

ridge. The same narrow ridge is found when neighboring energy bands are used to model the background. This is further proof that our concepts of background modeling and mosaicking of data spaces from individual observations appear to be applicable to diffuse emission.

The obvious question is whether the apparent differences between observations and simulations are indeed significant. This was studied by including the diffuse model in the likelihood search for sources, both for the observations and the simulations (for which only random fluctuations should show up). The bottom row of Figure 3 shows the resulting maps of $-2 \ln \lambda$, where $\lambda$ is now the maximum likelihood ratio $L$ (diffuse + background $) / L$ ( source + diffuse + background $)$. Indeed, the maps for the simulations are consistent with what
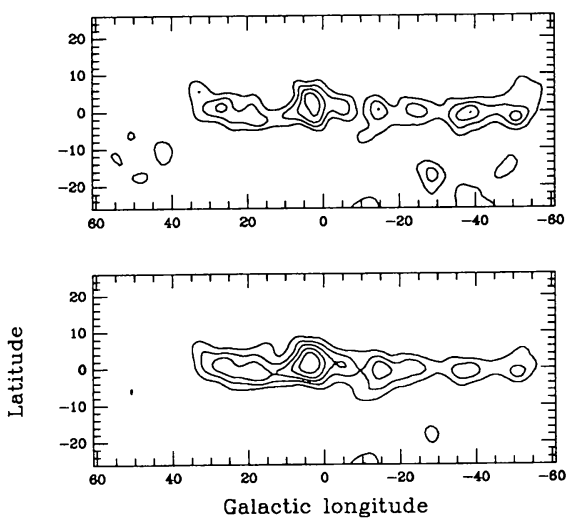

Fig. 4.-Maximum-entropy map (top) and likelihood map (bottom) of combined COMPTEL observations of the inner Galaxy in the $1.8 \mathrm{MeV}$ line of ${ }^{26} \mathrm{Al}$ (from Diehl et al. 1994). Note the very different global appearance of these maps (narrow ridge) compared to the observed continuum emission shown in Fig. 3. can be expected from statistics, but the observations show at least two clear excesses. A similar analysis of the EGRET data (Mayer-Hasselwander et al. 1994) shows three clear excesses in this region of which two are near the ones found here (the third EGRET source is at the Galactic center). The EGRET source near $l \approx 18^{\circ}$ is extended and coincides with a similar feature seen in the COS B data (Bloemen 1989) - it may be one of the most violent regions in the Galaxy. The EGRET source near $l \approx 343^{\circ}, b \approx-3^{\circ}$ originates from the $102 \mathrm{~ms}$ pulsar PSR 1706-44, uniquely identified through its pulsed signal. Also this source was seen in the $\operatorname{COS} B$ data $(2 \mathrm{CG}$ $342-02$ ). It is remarkable that both excess found here are $\sim 3^{\circ}$ further from the plane than the EGRET sources. This may indicate that we have overestimated the contribution from diffuse emission, which has shifted the sources away from the disk (we first fit the diffuse model and then search for unexplained excesses). On the other hand, so far no pulsed signal of PSR 1706-44 is found in the COMPTEL data, so the excess seen may originate from another source. The fact that only "sources" outside the Galactic disk are found may indicate that an ensemble of sources along the plane is hidden because we have overestimated the contribution from diffuse emission. This issue will be addressed in future work by fitting a number of sources simultaneously with diffuse emission.

There is clearly no evidence in the present analysis for the well-known hard $\mathrm{X}$-ray/low-energy $\gamma$-ray sources $1 \mathrm{E}$ 1740.7-2942 and GRS 1758-258 near the Galactic center. An excess found in the $0.75-1 \mathrm{MeV}$ band, however, coincides with 1E 1740.7-2942 (with a flux of $\sim 1 \times 10^{-4} \mathrm{~cm}^{-2} \mathrm{~s}^{-1}$, at least an order of magnitude above extrapolations of all published spectra of this source), but it cannot be excluded that it is actually due to diffuse emission (Bloemen et al. 1993).

We emphasize that this paper should mainly be regarded as a summary of our analysis strategy - the results are preliminary. 
Bertsch, D. L., \& Kniffen, D. A. 1983, ApJ, 270, 305

Blitz, L., et al. 1985, A\&A, 143, 267

Bloemen, H. 1987, ApJ, 317, L15 . 1989, ARA\&A, 27, 469.

- 1990, in ASP Conf. Ser. 12, The Evolution of the Interstellar Medium, ed. L. Blitz (San Francisco: ASP), 79

Bloemen, H., et al. 1993, Proc. Compton Science Symp., ed. M. Friedlander, N. Gehrels, \& D. J. Macomb (New York: AIP), 30

de Boer, H., et al. 1992, in Data Analysis in Astronomy IV, ed. V. Di Gesù et al. (New York: Plenum), 241

Dame. T. M., et al. 1987, ApJ, 322, 706

Diehl, R., et al. 1994, ApJS, 92, 429

Gehrels, N., et al. 1991, ApJ, 375, L13

Gehrels, N., \& Tueller, J. 1993, ApJ, 407, 597

Harris, M. J., et al. 1990, ApJ, 362, 135

\section{REFERENCES}

Hartman, R. C., et al. 1979, ApJ, 230, 597

Lavigne, J. M., et al. 1986, ApJ, 308, 370

Ling et al. 1987, ApJ, 321, L117

Mandrou, P., Bui-Van, A., Vedrenne, G., \& Niel, M. 1980, ApJ, 237, 424

Mayer-Hasselwander, H. A., et al. 1994, ApJS, in press

O’Neill, T. et al. 1983, Proc. 18th Internat. Cosmic-Ray Conf. (Bangelore), 9,45

Peterson, L. E., et al. 1990, Proc. 21 st Internat. Cosmic-Ray Conf. (Adelaide), 1,44

Riegler, G. R., et al. 1985, ApJ, 294, L13

Schönfelder, V., von Ballmoos, P., \& Diehl, R. 1988, ApJ, 335, 748

Schönfelder, V., et al. 1993, ApJS, 86, 657

Strong, A. W., et al. 1988, A\&A, 207, 1

1992, in Data Analysis in Astronomy IV, ed. V. Di Gesù et al. (New York: Plenum), 251 